\title{
Monte Carlo Simulations of Short-time Critical Dynamics with a Conserved Quantity
}

\author{
B. Zheng ${ }^{1,2}$ and H.J. Luo ${ }^{3}$ \\ ${ }^{1}$ FB Physik, Universität Halle, D-06099 Halle, Germany \\ ${ }^{2}$ Institute of Theoretical Physics, Academia Sinica, 100080 Beijing, P.R. China \\ ${ }^{3}$ FB Physik, Universität Siegen, D-57068 Siegen, Germany
}

\begin{abstract}
With Monte Carlo simulations, we investigate short-time critical dynamics of the three-dimensional anti-ferromagnetic Ising model with a globally conserved magnetization $m_{s}$ (not the order parameter). From the power law behavior of the staggered magnetization (the order parameter), its second moment and the auto-correlation, we determine all static and dynamic critical exponents as well as the critical temperature. The universality class of $m_{s}=0$ is the same as that without a conserved quantity, but the universality class of non-zero $m_{s}$ is different.
\end{abstract}

PACS: 64.60.Ht, 02.70.Lq, 75.10.Hk 


\section{INTRODUCTION}

About ten years ago, in a pioneering work by Janssen, Schaub and Schmittmann, shorttime universal scaling behavior of non-equilibrium critical dynamics starting from a high temperature state was systematically explored with renormalization group methods [1]. In a few years, extension of the calculations to different critical dynamics was carried out by Oerding and Janssen in a series of works [2, 24. Some evidences for the short-time dynamic scaling were also observed in Monte Carlo simulations [5]. Meanwhile, it was found that the power law decay of the magnetization starting from a completely ordered state emerges already in relatively early times, e.g., see [8], and it can be used to estimate the dynamic exponent $z$ [9.10]. In recent years short-time critical dynamics has been systematically investigated with Monte Carlo simulations [11]15]. Simulations have extended from regular classical spin models [14,16 19] to statistical systems with quenched disorder [20 22], quantum spin systems [23], lattice gauge theories [24], the hard-disk model [25],26] and dynamic systems without detailed balance [27 31]. The references given here are only a part of recent ones and not complete. A relatively complete list of the relevant references before 1998 can be found in a recent review, Ref. [14]. All numerical and analytical results confirm that there exists a rather general dynamic scaling form in critical dynamic systems already in the short-time regime of dynamic evolution.

Traditionally, it is believed that the short-time behavior of dynamic systems depends essentially on microscopic details. To understand short-time universal behavior, one should distinguish two different time scales, the macroscopic and microscopic time scales. The short-time dynamic scaling form emerges only after a time scale $t_{m i c}$, which is sufficiently large in microscopic sense, but still very small in macroscopic sense. $t_{m i c}$ is a time that the system needs to sweep away the effect of microscopic details. In Monte Carlo simulations, for example, if a sweep over all spins on a lattice is considered to be a microscopic time unit, $t_{m i c}$ is usually the order of 10 to 100 Monte Carlo time steps [14]. For the simple Ising and Potts models with only nearest neighbor interactions, sometimes $t_{m i c}$ is negligible small (e.g., one Monte Carlo time step or two). Compared with typical macroscopic time scales characterized by $\tau^{-\nu z}$ or $L^{z}$ around the critical point, $t_{m i c}$ is indeed very small.

The physical origin of the short-time dynamic scaling is the divergent correlation time near a critical point. The divergent correlation time induces a memory effect, and the memory effect can be described by a scaling form. How does this scaling form look like? As pointed out by Janssen, Schaub and Schmittmann [1], one should introduce new critical exponents to describe the dependence of the scaling behavior on the macroscopic initial conditions. Actually, for arbitrary initial conditions even a characteristic function is needed 32,33. Furthermore, to describe the scaling behavior of some special dynamic observables, we also have to introduce new exponents, for example, the persistence exponent [34, 35.

The short-time dynamic scaling form is not only conceptually interesting but also practically important. It provides new techniques for the measurements of both dynamic and static critical exponents as well as the critical temperature, for a review, see Ref. [14]. Since the measurements are carried out in the short-time regime of dynamic evolution, the dy- 
namic approach does not suffer from critical slowing down. A Averaging is over both initial configurations and the random forces. This is very different from the time averaging in the measurements in equilibrium. Compared with those non-local methods which are invented to overcome the critical slowing down in Monte Carlo simulations in equilibrium, e.g., the cluster algorithms, the dynamic approach does study the original local dynamics and can be applied to disordered systems.

In fact, the dynamic scaling in non-equilibrium critical dynamics is not an exclusive phenomenon in the world. In many other non-equilibrium dynamic processes, universal or quasi-universal scaling behavior has been also observed. An example is phase ordering dynamics [36. In this case, a real equilibrium state and a macroscopic time scale like $\tau^{-\nu z}$ do not exist. Therefore, 'short-time' is not addressed. Another example is aging in complex systems such as glasses and spin glasses. Aging is just a kind of scaling or quasiscaling. Dynamic scaling behavior around a spin glass transition [5, 37, 39,21, is very similar to that around a standard critical point. For example, the experimental measurements of the remanent magnetization in spin glasses support not only the power law scaling behavior but also the scaling relations between the exponents [40,21].

After such a slightly lengthy review, we come to what we are going to do in this paper. Even though renormalization group calculations have been extended to different critical dynamics, up to now, Monte Carlo simulations are limited in dynamics of model A 41. Actually, due to severe critical slowing down, Monte Carlo simulations for long-time behavior of critical dynamics beyond model A are also in the preliminary stage. The short-time dynamic approach provides powerful methods for numerical measurements of not only dynamic exponents, but also static exponents as well as the critical temperature. Simulations of dynamic systems beyond model A in equilibrium are usually difficult. Therefore, it is important to investigate short-time scaling behavior of these dynamic systems with Monte Carlo methods.

Dynamics of model A is a kind of relaxational dynamics without relevant conserved quantities 41]. If we consider only dynamic relaxational processes, dynamics with different relevant conserved quantities is classified into model $\mathrm{B}, \mathrm{C}$ and $\mathrm{D}$. In this paper, taking the three-dimensional anti-ferromagnetic Ising model for example, with Monte Carlo methods we study dynamics with a conserved quantity, which is not an order parameter but coupling to the order parameter. According to Ref. [41], this is called model C. In the next section, we introduce the model and analyze the power law scaling behavior in the short-time regime. In section 3, we present the numerical results. Finally come the concluding remarks.

\section{SHORT-TIME DYNAMIC SCALING}

\footnotetext{
${ }^{1}$ In the literature, sometimes it is stated that the short-time dynamic approach can eliminate critical slowing down. Rigorously speaking, this statement is not correct. Critical slowing down always exists, at least for local dynamics, and it is the physical origin of the dynamic scaling. But in the short-time dynamic approach we do not have the problem of generating independent configurations. Therefore, we do not suffer from critical slowing down.
} 


\section{A. The model}

We consider an antiferromagnetic Ising model on a three-dimensional cubic lattice. The Hamiltonian of the model is

$$
-H / k T=-K \sum_{<i j>} S_{i} S_{j}
$$

where $S_{i}$ is an Ising spin and the sum is over nearest neighbors. For dynamics of model $\mathrm{A}$ and in equilibrium, on a cubic lattice the antiferromagnetic Ising model is equivalent to the (ferromagnetic) Ising model. Now, we keep the magnetization as a constant in dynamic evolution. Here the order parameter is not the magnetization but the staggered magnetization. We will denote the magnetization by $m_{s}$ and the staggered magnetization by $M(t)$. For equilibrium states, it is expected that for $m_{s}=0$, the universality class is the same as that of the standard Ising model without a conserved magnetization. For a non-zero $m_{s}$, according to Ref. [41], the critical exponent $\nu$ is different from that of $m_{s}=0.0$, but it does not depend on the value of the non-zero $m_{s}$. For critical dynamics, the dynamic exponent $z$ and the exponent $\theta$ for $m_{s}=0$ are the same as those of the standard Ising model, but for non-zero $m_{s}$ they are different. Again, $z$ and $\theta$ do not depend on the value of the non-zero $m_{s}$. These conclusions are drawn from renormalization group calculations based on the $\phi^{4}$ theory coupling to a conserved current 441,2]. It is interesting to compared these results with Monte Carlo simulations.

In this paper, we study only dynamic relaxation starting from disordered states with a zero or small initial staggered magnetization $m_{0}$. We measure how the staggered magnetization and its second moment as well as the auto-correlation evolve in the dynamic process. The heat-bath algorithm is used in simulations. In order to keep $m_{s}$ as a constant, in a flip we simply exchange the values of two spins. Here we should point out that if our model is not the anti-ferromagnetic but the ferromagnetic Ising model, the (conserved) magnetization $m_{s}$ is the order parameter and then the dynamics for $m_{s}=0$ belongs to model B 441.

In dynamics without any relevant conserved quantities, i.e., dynamics of model A, updating schemes are irrelevant in the sense of universality. Different updating schemes lead to the same critical exponents, either in an equilibrium or a non-equilibrium state. However, for dynamics with relevant conserved quantities, it is different, at least for non-equilibrium short-time behavior. In a recent work by one of the authors [42], for example, the Monte Carlo dynamics with a conserved order parameter for the two-dimensional Ising model is investigated. It is found that if in a flip we exchange the values of two neighboring spins, the dynamics is very slow. However, if we release the condition such that the order parameter is not locally conserved but only globally conserved (for example, in a flip we exchange the values of two randomly separated spins), the dynamic exponent $z$ is much smaller. For the dynamics studied in this paper, i.e., with a conserved quantity which is not the order parameter, the situation is somewhat similar. If in a flip we exchange the values of two neighboring spins, dynamic evolution is somewhat slow. Therefore, as a first approach, we decide to keep the magnetization $m_{s}$ only globally conserved. In a flip we exchange the values of two randomly separated spins. Except for the updating schemes, all computational techniques adopted here are the same as those in the simulations of model A [14]. 


\section{B. Short-time dynamic scaling}

For critical dynamic systems, traditionally it is believed that universal scaling behavior exists only in the long-time regime of dynamic evolution. However, in recent years it is discovered that starting from macroscopic initial states, universal scaling behavior emerges already in the macroscopic short-time regime of dynamic processes after a microscopic time scale $t_{m i c}$ [1, 5, 6, 11, 12, 14]. A typical example is that a magnetic system initially in a high temperature state with a small initial order parameter $m_{0}$, is suddenly quenched to the critical temperature $T_{c}$ or nearby (without external magnetic field) and then released to dynamic evolution of model A [41]. A generalized dynamic scaling form can be written down, for example, for the $k$ th moment of the order parameter,

$$
M^{(k)}\left(t, \tau, L, m_{0}\right)=b^{-k \beta / \nu} M^{(k)}\left(b^{-z} t, b^{1 / \nu} \tau, b^{-1} L, b^{x_{0}} m_{0}\right) .
$$

Here $t$ is the time variable, $\tau$ is the reduced temperature, $L$ is the lattice size. $\beta$ and $\nu$ are standard static exponents and $z$ is the dynamic exponent. Important is that a new independent exponent $x_{0}$ is introduced to describe the scaling behavior of the initial order parameter $m_{0}$. If the scaling form above is valid, all relevant exponents can be extracted from the short-time behavior of relevant observables.

For the dynamic system with a conserved quantity discussed in the last subsection, we assume that a scaling form like Eq. (2) holds also. In this case, $\tau=\left(K-K_{c}\right) / K_{c}$ and $K_{c}$ depends on the conserved magnetization $m_{s}$, i.e., there is a critical line $K_{c}=K_{c}\left(m_{s}\right)$. In principle, there are two possibilities for the critical exponents. In the case of strong universality, the value of an exponent for non-zero $m_{s}$ can be different from that for zero $m_{s}$, but does not depend on the value of the non-zero $m_{s}$. In the case of weak universality, an exponent may vary its value continuously along a critical line. For the $\phi^{4}$ theory coupling to a conserved current in Ref. [41], it is the case of strong universality.

Neglecting the finite size effect and noting that $m_{0}$ is small, we expand the right hand side of Eq. (2) and take only the first non-zero (linear) term under the condition of a small $t$. At the initial stage of time evolution, the staggered magnetization around the critical temperature behaves as,

$$
M\left(t, \tau, m_{0}\right) \sim m_{0} t^{\theta} F\left(t^{1 / \nu z} \tau\right), \quad \theta=\left(x_{0}-\beta / \nu\right) / z .
$$

At the exact critical point, $\tau=0, M(t)$ obeys a power law $\sim t^{\theta}$. Numerical results and analytical calculations have revealed that the exponent $\theta$ is positive for almost all systems, i.e., the order parameter undergoes an initial increase. This makes the short-time behavior very prominent. The physical mechanism for this increase has not been very clear. At least the mean-field effect or symmetry breaking is not very relevant. Slightly away from the critical point, the power law behavior is modified by the scaling function $F\left(t^{1 / \nu z} \tau\right)$. This fact allows us to locate the critical temperature and meanwhile to measure the critical exponent $\theta$.

Differentiation of Eq. (3) leads to

$$
\left.\partial_{\tau} M\left(t, \tau, m_{0}\right)\right|_{\tau=0}=\left.m_{0} t^{c_{d}} \partial_{\tau^{\prime}} F\left(\tau^{\prime}\right)\right|_{\tau^{\prime}=0}, \quad c_{d}=1 / \nu z+\theta .
$$

From this power law behavior, one can determine the critical exponent $1 / \nu z$. 
Taking into account that the non-equilibrium spatial correlation length $\left(\sim t^{1 / z}\right)$ is small at the initial stage of time evolution, from Eq. (2) it can be derived that the second moment at $T_{c}$ subjects to a finite size scaling

$$
M^{(2)}(t, L) \sim L^{-d} t^{y}, \quad y=(d-2 \beta / \nu) / z
$$

For simplicity, here we have set $m_{0}=0$. Another interesting dynamic observable is the auto-correlation

$$
A(t) \equiv \frac{1}{L^{d}}\left\langle\sum_{i} S_{i}(0) S_{i}(t)\right\rangle .
$$

At the critical temperature $T_{c}, A(t)$ decays by a power law 43

$$
A(t) \sim t^{-\lambda}, \quad \lambda=\frac{d}{z}-\theta .
$$

It is interesting that even though we have set $m_{0}=0, \theta$ (i.e., $x_{0}$ ) still enters the autocorrelation. This is because $x_{0}$ is actually the scaling dimension of the local order parameter.

From Eqs. (5) and (7), we are able to estimate the static exponent $\beta / \nu$ and the dynamic exponent $z$. Then we complete the measurements of all the exponents and the critical temperature. For details of the above scaling analysis and a more systematic extension, readers are referred to Refs. [1,43] and the recent review article Ref. [14].

\section{MONTE CARLO SIMULATIONS}

$$
\text { A. } m_{s}=0
$$

For the anti-ferromagnetic Ising model, in equilibrium the averaged magnetization is zero. If we notice that the order parameter is the staggered magnetization, the thermodynamic fluctuation of the magnetization goes to zero in the thermodynamic limit (infinite volume). In non-equilibrium dynamic processes without any conserved quantities, if one starts from initial states with a zero magnetization $m_{s}$, the magnetization will remain zero. This is the case for usual macroscopic initial states, e.g., the high temperature and low temperature states. The fluctuation of the magnetization in the dynamic processes is also zero in thermodynamic limit. Therefore, one expects that the equilibrium state of the dynamic process with a conserved magnetization $m_{s}=0$ is in a same universality class of the model without a conserved quantity, and so is the dynamic universality class as well. The critical temperature is also not changed.

We perform simulations for $m_{s}=0$ in order to confirm the above expectation and to demonstrate our computational techniques. The heat-bath algorithm is adopted in simulations since it is faster than the Metropolis algorithm in the short-time regime. Lattice sizes are $L=64$ and 128. Within our maximum updating times, no finite size effects are observed. We remind the readers here that it is an advantage of the short-time dynamic approach that the finite size effects are easily controlled due to the small non-equilibrium spatial correlation length $\left(\sim t^{1 / z}\right)$. 
The critical temperature has been measured rather accurately in simulations in equilibrium (for the system without a conserved quantity). However, we also present data to determine it from short-time dynamics. With the short-time dynamic approach, in principle, any observables which are sensitive to the temperature around the critical regime can be used for the determination of the critical temperature.

In Fig. 1, time evolution of the staggered magnetization $M(t)$ is displayed with solid lines in $\log$ - $\log$ scale. The lattice size is $L=128$, and 2500 samples of initial configurations have been used for averaging. In order to see the possible 'finite $m_{0}$ effects', we have performed the simulations with $m_{0}=0.01$ and 0.02 . The coupling constant $K$ is $0.22065,0.22165$ and 0.22265 (from below) for $m_{0}=0.01$, and $0.22115,0.22165$ and 0.22215 for $m_{0}=0.02$. In principle, the closer $K$ 's are used, the more accurate $K_{c}$ and the critical exponent $1 / \nu z$ can be obtained. However, we suffer from large statistical fluctuation if the $K$ 's are too close to each other. We use different $K$ 's for $m_{0}=0.01$ and $m_{0}=0.02$ just to study the possible systematic errors.

To make use of Eq. (3) to locate the critical point, we first interpolate $M(t)$ quadratically to any $K$ around the three simulated ones, then search for a $K$ which gives the best power law behavior for the curve. This $K$ is $K_{c}$. The two dashed lines in Fig. 11 are the curves with the best power law behavior for $m_{0}=0.01$ and $m_{0}=0.02$. The corresponding $K_{c}$ 's are $K_{c}=0.22169(9)$ and $0.22163(5)$ for $m_{0}=0.01$ and $m_{0}=0.02$ respectively. These two values are consistent each other and in agreement with that of the Ising model measured in equilibrium (without any conserved quantity), which is reported to be around 0.22165 44,.45]. We perform our measurements in a time interval $\left[t_{m i c}, 400\right]$. The results are rather stable when we take $t_{m i c}$ bigger than 10.

With $K_{c}$ in hand, we measure the critical exponent $\theta=0.108(5)$ and $0.100(5)$ for $m_{0}=$ 0.01 and $m_{0}=0.02$. They are consistent with $\theta=0.104(3)$ in Ref. [13] and $\theta=0.108(2)$ in Ref. [46] for the Ising model without a conserved quantity. Rigorously speaking, the exponent $\theta$ is defined in the limit of $m_{0}=0$. We should extrapolate $\theta$ to $m_{0}=0$. However, since here there are some statistical errors, and especially the errors induced by the errors of the critical point $K_{c}$ 's, it is not meaningful to do so. To reduce the errors induced by $K_{c}$, we could take $K_{c}=0.22165$ as input. Then we obtain $\theta=0.106(6)$ by extrapolating the results to $m_{0}=0$. In this paper, we simply consider $\theta=0.108(5)$ measured from $m_{0}=0.01$ as our final value of $\theta$.

In Fig. 2, time evolution of the second moment of the staggered magnetization is displayed with solid lines in log-log scale. Here the initial staggered magnetization $m_{0}$ has been set to zero. From below, $K=0.22065,0.22165$ and 0.22265 . The lattice size is $L=64$ and the number of samples for averaging is 3200 . Extra simulations for $L=128$ show that the finite size effect for $L=64$ is already negligible small. From the figure, we see that the second moment is apparently less sensitive to $K$, compared with the staggered magnetization itself. However, we still can locate the critical $K_{c}$ from the data. Measuring in a time interval $\left[t_{m i c}=20,700\right], K_{c}=0.22164(22)$. The curve corresponding to $K_{c}$ is shown by a dashed line in the figure. The error of $K_{c}$ here is bigger than that from the staggered magnetization. To reduce the error, simply increasing the samples for averaging is not enough, one must have longer maximum updating times and therefore larger lattices. These need much CPU times since the second moment is not self-averaging. To estimate the exponent $y=(d-2 \beta / \nu) / z$, for simplicity, we take $K_{c}=0.22165$ as input. The result is $y=0.965(11)$. 
In the simulations for Fig. 2, we have also measured the auto-correlation function $A(t)$. The results are shown in Fig. 包. From below, $K=0.22065,0.22165$ and 0.22265 . It is impossible to locate the critical point from the auto-correlation. We measure the exponent $\lambda=1.36(3)$ at $K_{c}=0.22165$ in a time interval $[20,100]$. When $t$ is bigger than 100 , the fluctuation becomes large.

In order to determine the critical exponent $\nu$, we need $\left.\partial_{\tau} M(t, \tau)\right|_{\tau=0}$. From the data for

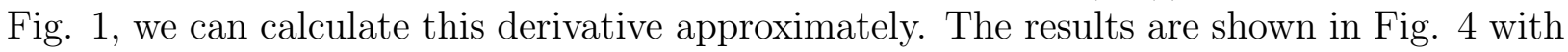
solid lines in log-log scale. From below, the initial staggered magnetization is $m_{0}=0.01$ and 0.02. It is known that the power law behavior of the derivative of $M(t)$ in shorter times is less clean than that of $M(t)$ itself [14]. Therefore, we measure the slopes in a time interval $[70,400]$ and obtain $c_{d}=1 / \nu z+\theta=0.867(10)$ and $0.901(22)$ for $m_{0}=0.01$ and 0.02 respectively. The value from $m_{0}=0.01$ is more reliable.

In Table @, we list all the exponents we have measured. Taking the exponent $\theta$ as input, from $\lambda$ we estimate the dynamic exponent $z$. Then from $y$ and $c_{d}$ we calculate the exponents $2 \beta / \nu$ and $\nu$. For comparison, available results for dynamics without a conserved quantity [46], i.e., the so-called model A dynamics, are also given, where the critical point $K_{c}$ and the exponent $\nu$ are measured from another dynamic process starting from a completely ordered state. Within statistical errors, all the exponents for dynamics with and without a conserved quantity agree well each other. The static exponents and $K_{c}$ are also consistent with those of the standard Ising model measured from simulations in equilibrium with the cluster algorithms [45]. Here we should make a comment. Even for dynamics of model A, the static exponents and $K_{c}$ measured from the short-time dynamics are still not as accurate as those obtained with the cluster algorithms. One reason is that the best effort for the short-time dynamic approach has not been made. Another reason is that in the short-time dynamic approach we have to measure both the static and dynamic exponents together. It makes the work more difficult than measuring only the static exponents. However, it is clear if we are interested in both static and dynamic properties, the short-time dynamic approach is very efficient. The cluster algorithms are non-local and change already the dynamic universality class. On the other hand, the cluster algorithms can not straightforwardly apply to any systems, e.g., the systems with quenched randomness and lattice gauge theories.

\section{B. $m_{s} \neq 0$}

Encouraged by the success for $m_{s}=0.0$, we proceed to $m_{s} \neq 0$. The results in the last subsection show that the lattices sizes $L=64$ and 128 are sufficient for our maximum updating times. The finite $m_{0}$ effect for $m_{0}=0.01$ is already invisible within the statistical errors. Therefore, in this subsection we will not systematically study the finite size and finite $m_{0}$ effects. However we have also performed some extra calculations to confirm the results presented. To locate the critical point $K_{c}$ and estimate the exponent $\nu$, we need data for several $K$ 's in the neighborhood of the critical point. The results in the last subsection also show that the difference of these $K$ 's should be around half a percent of $K_{c}$ or less.

We perform simulations for $m_{s}=0.2$ and 0.4. For $m_{s}=0.2$ we obtain data for $K=$ $0.2405,0.2410,0.2415$ and 0.2420 , which are shown in Fig. 5 with solid lines (from below) in $\log -\log$ scale. For $m_{s}=0.4$ we obtain data for $K=0.3310,0.3320,0.3330$ and 0.3340 , 
which are shown in Fig. 6 with solid lines (from below) in log-log scale. The lattice size is $L=128$ and 2500 samples of the initial configurations are used for averaging.

Following the procedure in the last subsection, searching for a curve with the best power law behavior in a time interval $\left[t_{m i c}, 400\right]$, one can determine $K_{c}$ and the critical exponent $\theta$. Now we have data for four $K$ 's and therefore have the choices to interpolate the staggered magnetization with three or four $K$ 's. For $K_{c}$, both yield the same values within errors. With four $K$ 's, we obtain $K_{c}=0.24153(12)$ for $m_{s}=0.2$ and $K_{c}=0.33230(20)$ for $m_{s}=0.4$ with $t_{m i c}=20$. The corresponding curves are shown in Figs. 5 and 6 with dashed lines. For $m_{s}=0.4, K_{c}$ is very stable for different choices of $t_{m i c}$. For $m_{s}=0.2, K_{c}$ increases slightly when we take bigger $t_{m i c}$. The reason is that our maximum updating times are not long enough. After carefully analyzing the data and looking at the curves in the figure, we convince ourself that $K_{c}=0.24153(12)$ is the most reasonable.

In Fig. 4, $\left.\partial_{\tau} M(t, \tau)\right|_{\tau=0}$ is displayed with dashed lines for $m_{s}=0.2$ and 0.4 (from above). The results are obtained with four $K$ 's. For $m_{s}=0.4$, the resulting exponent $\nu$ is bad with three $K$ 's. The reason is clear. $K_{c}$ is between $K=0.3320$ and 0.3330 . If we choose only three $K$ 's, we have some systematic errors in interpolation.

With $K_{c}$ in hand, we perform simulations for $m_{0}=0.0$ and measure the second moment and the auto-correlation. A lattice size $L=64$ is used and the number of samples of initial configuration for averaging is 7500. In Fig. 7, the second moment is displayed in log-log scale with solid lines for $m_{s}=0.2$ and $m_{s}=0.4$ (from above). For comparison, the second moment for $m_{s}=0.0$ is also plotted with a dashed line in the figure. Apparently, all three curves are parallel each other. The exponent $y$ is independent of $m_{s}$. In Fig. 8, the autocorrelation is displayed in log-log scale with solid lines for $m_{s}=0.2$ and $m_{s}=0.4$ (from below) and with a dashes line for $m_{s}=0.0$. In all the relevant figures for non-zero $m_{s}$, nice power law behavior is observed.

In Table @, all the exponents are summarized. Within the statistical errors, the static exponent $2 \beta / \nu$ is independent of $m_{s}$. The values also coincide well with those obtained from dynamics of model $\mathrm{A}$ and measured in equilibrium for the standard Ising model. The static exponent $\nu$ for $m_{s}=0.2$ and 0.4 are $0.82(8)$ and $0.81(3)$ respectively. Even though the first value carries a relatively big error, they are clearly different from $\nu=0.64(2)$ for $m_{s}=0.0$ and those measured from dynamics of model $\mathrm{A}$ and in equilibrium. However, $\nu$ is independent of the value of the non-zero $m_{s}$. These results are consistent with the theoretical calculations based on a $\phi^{4}$ theory coupling to a conserved current 41]. With our accuracy, we can not detect any dependence of the dynamic exponent $z$ on $m_{s}$. This differs from the $\phi^{4}$ theory in Ref. [41]. The exponent $\theta$ is $0.129(5)$ and $0.148(4)$ for $m_{s}=0.2$ and 0.4 respectively . These two values are obviously different from $\theta=0.108(5)$ for $m_{s}=0.0$, and show the nontrivial dynamic behavior for nonzero $m_{s}$. However, with our data we should conclude that $\theta$ depends on the value of the non-zero $m_{s}$, even though there may be still some uncontrolled systematic errors. This point is different from the $\phi^{4}$ theory.

\section{CONCLUDING REMARKS}

We have reported our Monte Carlo simulations of short-time critical dynamics for the three-dimensional anti-ferromagnetic Ising model with a globally conserved magnetization (not an order parameter). The power law scaling behavior has been observed for the stag- 
gered magnetization (the order parameter), its second moment and the auto-correlation. All dynamic and static critical exponents are determined. For a conserved magnetization $m_{s}=0.0$, all the exponents are the same as those of model A. For a non-zero $m_{s}$, the static exponents behave qualitatively the same as those of the $\phi^{4}$ theory coupling to a conserved current [41]. However, the dynamic exponents are somewhat different. The reason might be that the magnetization is not locally but only globally conserved. Therefore, it is interesting to study the dynamics, with a locally conserved order parameter or non-order parameter: in a flip, we exchange two spins in a local regime, the size of which must be much smaller than the lattice size.

Acknowledgements: B.Z. thanks deeply Oerding for suggesting the topic and inspiring discussions. This work is supported in part by DFG; TR 300/3-1 and Schu 95/9-2. 


\section{REFERENCES}

[1] H. K. Janssen, B. Schaub and B. Schmittmann, Z. Phys. B 73, 539 (1989).

[2] K. Oerding and H. K. Janssen, J. Phys. A26, 3369,5295 (1993).

[3] K. Oerding and H. K. Janssen, J. Phys. A27, 715 (1994).

[4] K. Oerding and H. K. Janssen, J. Phys. A28, 4271 (1995).

[5] D. A. Huse, Phys. Rev. B 40, 304 (1989).

[6] K. Humayun and A. J. Bray, J. Phys. A24, 1915 (1991).

[7] N. Menyhárd, J. Phys. A27, 663 (1994).

[8] D. Stauffer, Physica A 186, 197 (1992).

[9] C. Münkel, D. W. Heermann, J. Adler, M. Gofman and D. Stauffer, Physica A 193, 540 (1993).

[10] N. Ito, Physica A 196, 591 (1993).

[11] Z.B. Li, U. Ritschel and B. Zheng, J. Phys. A27, L837 (1994).

[12] L. Schülke and B. Zheng, Phys. Lett. A 204, 295 (1995).

[13] P. Grassberger, Physica A 214, 547 (1995).

[14] B. Zheng, Int. J. Mod. Phys. B12, 1419 (1998), review article.

[15] B. Zheng, M. Schulz and S. Trimper, Phys. Rev. Lett. 82, 1891 (1999).

[16] J. B. Zhang, L. Wang, D. W. Gu, H. P. Ying and D. R. Ji, Phys. Lett. A262, 226 (1999).

[17] B. Zheng, M. Schulz and S. Trimper, Phys. Rev. E59, R1351 (1999).

[18] A. J. Bray, A. J. Briant and D. K. Jervis, Phys. Rev. Lett. 84, 1503 (2000).

[19] L. M. Jensen, B. J. Kim and P. Minnhagen, Phys. Rev. B61, 15412 (2000).

[20] H.J. Luo, L. Schülke and B. Zheng, Phys. Rev. Lett. 81, 180 (1998).

[21] H.J. Luo, L. Schülke and B. Zheng, Mod. Phys. Lett. B13, 417 (1999).

[22] H. P. Ying and K. Harada, Phys. Rev. E62, 174 (2000).

[23] H.P. Ying, H.J. Luo, L. Schülke, B. Zheng, Mod. Phys. Lett. B12, 1237 (1998).

[24] K. Okano, L. Schülke and B. Zheng, Phys. Rev. D57, 1411 (1998).

[25] A. Jaster, Phys. Lett. A258, 59 (1999).

[26] A. Jaster, Physica A277, 106 (2000).

[27] N. Menyhárd and G. Ódor, J. Phys. A29, 7739 (1996).

[28] J. F. F. Mendes and M. A. Santos, Phys. Rev. E57, 108 (1998).

[29] T. Tomé and J. R. D. de Felício, Mod. Phys. Lett. B12, 873 (1998).

[30] T. Tomé and M. J. de Oliveira, Phys. Rev. E58, 4242 (1998).

[31] A. Brunstein and T. Tomé, Phys. Rev. E60, 3666 (1999).

[32] B. Zheng, Phys. Rev. Lett. 77, 679 (1996).

[33] Y. Chen, S. H. Guo, Z. B. Li and A. Ye, Euro. Phys. J. B B15, 97 (2000).

[34] S.N. Majumdar, A.J. Bray, S. Cornell and C. Sire, Phys. Rev. Lett. 77, 3704 (1996).

[35] L. Schülke and B. Zheng, Phys. Lett. A 233, 93 (1997).

[36] A. J. Bray, Adv. Phys. 43, 357 (1994), and references therein.

[37] K. Fischer and J. Hertz, Spin Glasses (Cambridge University Press, Cambridge, 1991).

[38] R. E. Blundell, K. Humayun and A. J. Bray, J. Phys. A25, L733 (1992).

[39] J. Kisker, L. Santen, M. Schreckenberg and H. Rieger, Europhys. Lett. 20, 159 (1992).

[40] P. Granberg, P. Svedlindh, P. Nordblad, L. Lundgren and H. S. Chen, Phys. Rev. B35, 2075 (1987).

[41] P.C. Hohenberg and B.I. Halperin, Rev. Mod. Phys. 49, 435 (1977). 
[42] B. Zheng, preprint, cond-mat/0103133.

[43] H. K. Janssen, in From Phase Transition to Chaos, edited by G. Györgyi, I. Kondor, L. Sasvári and T. Tél, Topics in Modern Statistical Physics (World Scientific, Singapore, 1992).

[44] A. M. Ferrenberg and D. P. Landau, Phys. Rev. B 44, 5081 (1991).

[45] H. W. J. Blöte, E. Luijten and J. R. Heringa, J. Phys. A28, 6289 (1995).

[46] A. Jaster, J. Mainville, L. Schülke and B. Zheng, J. Phys. A32, 1395 (1999). 


\section{FIGURES}

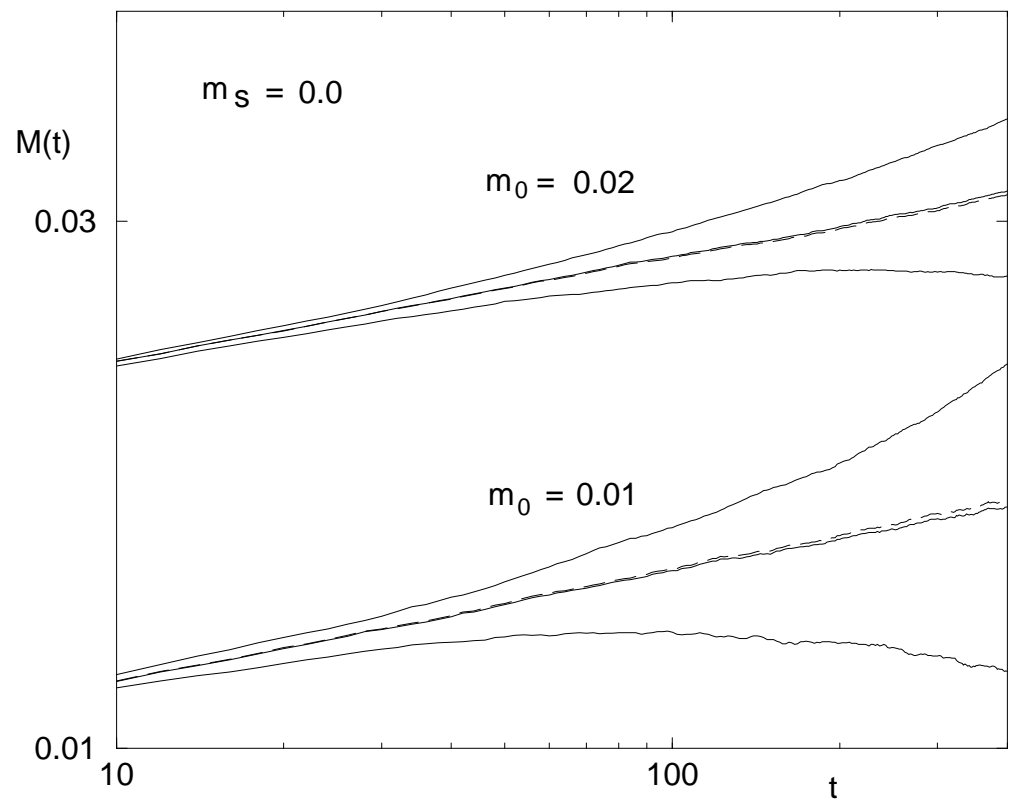

FIG. 1. Time evolution of the staggered magnetization displayed in log-log scale. For solid lines, $K$ is $0.22065,0.22165$ and 0.22265 (from below) for $m_{0}=0.01$, and $K$ is $0.22115,0.22165$ and 0.22215 for $m_{0}=0.02$. Dashed lines correspond to $K_{c}=0.22169(9)$ and $0.22163(5)$ for $m_{0}=0.01$ and $m_{0}=0.02$. 


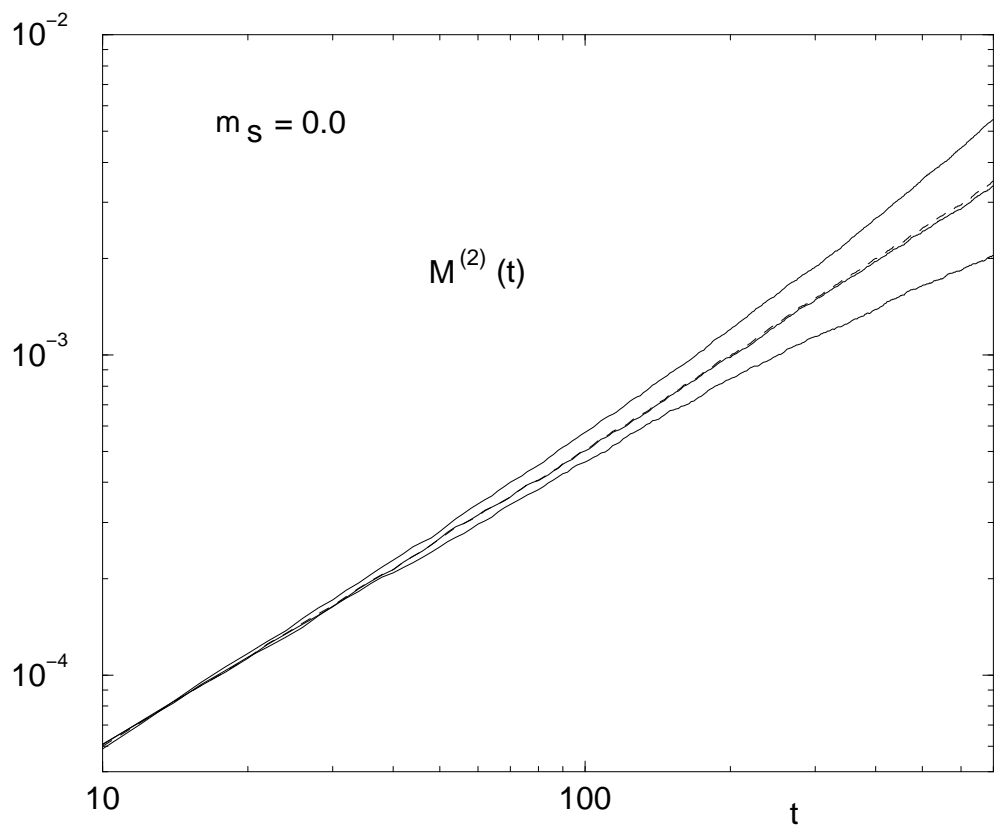

FIG. 2. The second moment in log-log scale. For solid lines, $K$ is $0.22065,0.22165$ and 0.22265 (from below). The dashed line corresponds to $K_{c}=0.22164(22)$. 


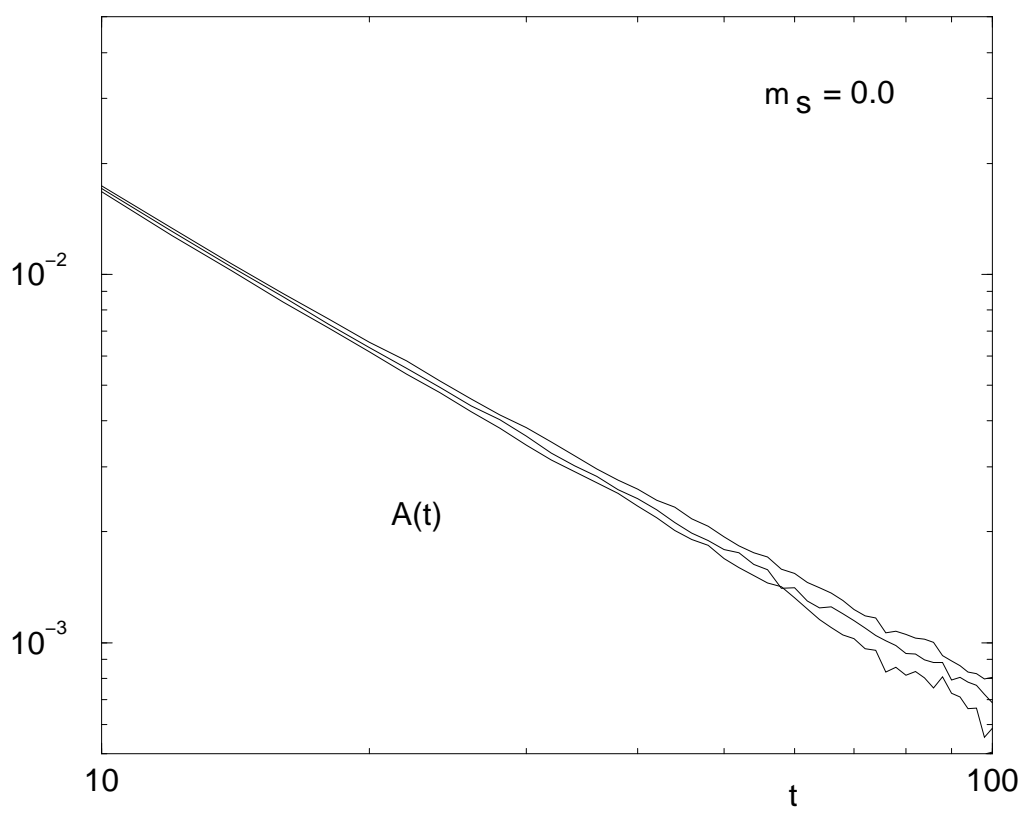

FIG. 3. The auto-correlation in log-log scale. $K$ is $0.22065,0.22165$ and 0.22265 (from below). 


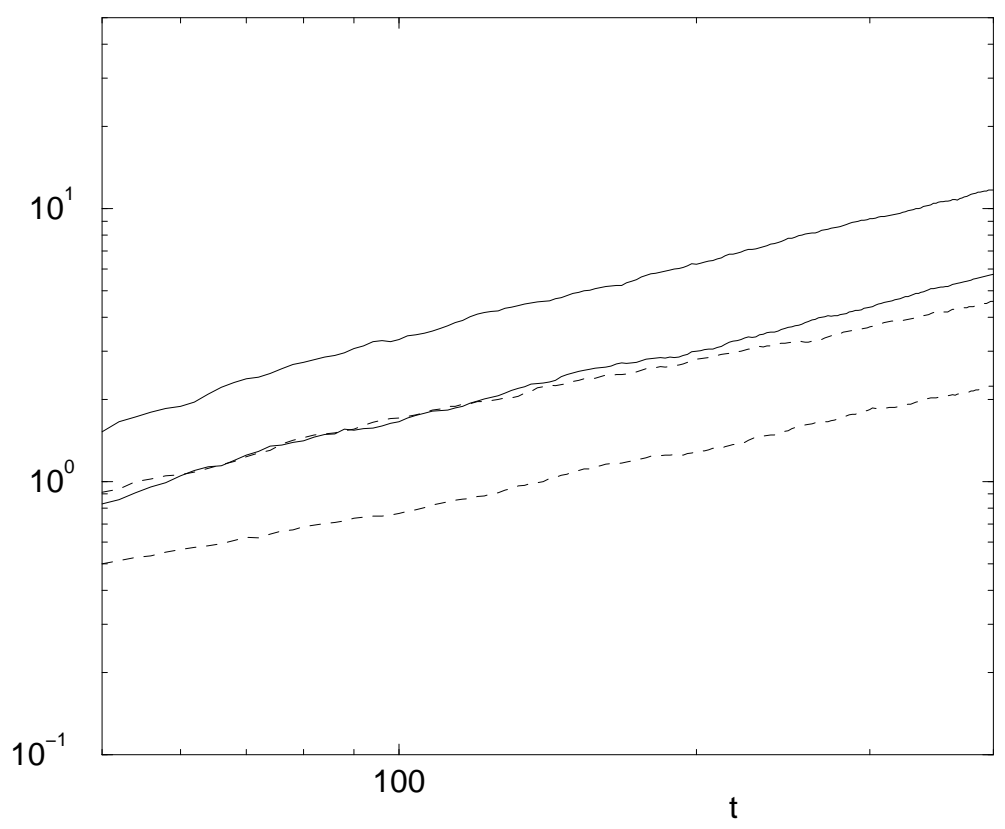

FIG. 4. The derivative $\left.\partial_{\tau} M(t, \tau)\right|_{\tau=0}$ in log-log scale. Solid lines are for $m_{s}=0.0$ and from below, $m_{0}=0.01$ and 0.02. Dashed lines are for $m_{s}=0.2$ and 0.4 (from above) with $m_{0}=0.01$. 


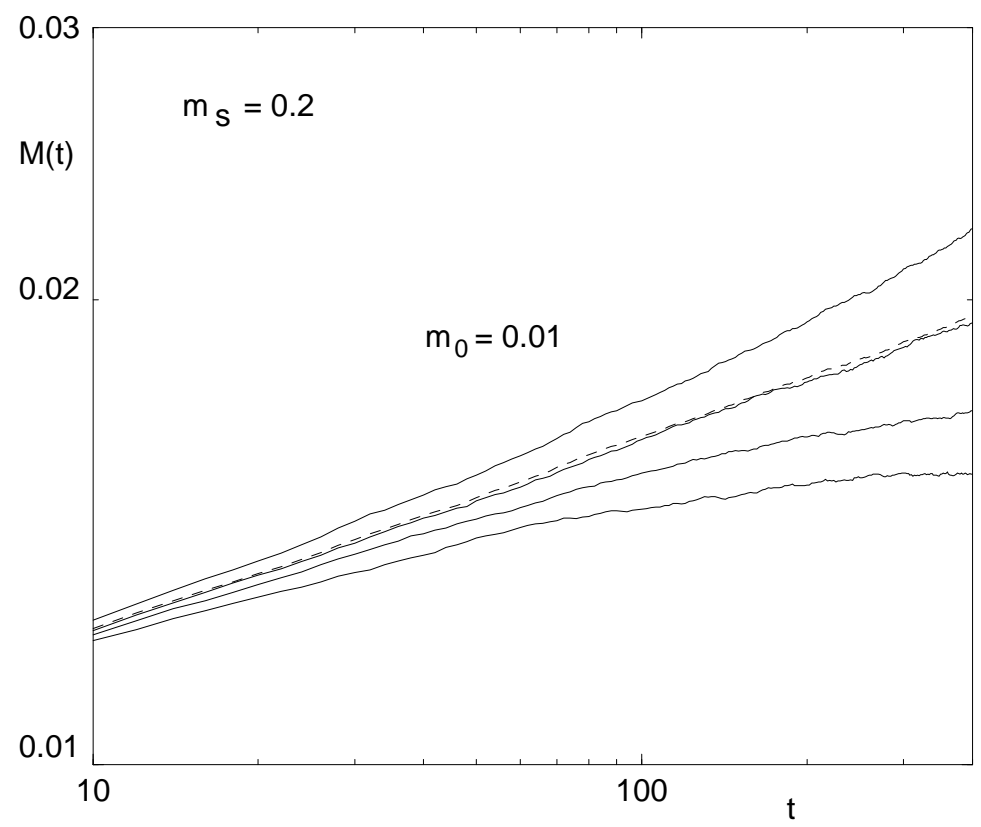

FIG. 5. Time evolution of the staggered magnetization for $m_{s}=0.2$ in log-log scale . For solid lines, $K$ is $0.2405,0.2410,0.2415$ and 0.2420 (from below). The dashed line corresponds to $K_{c}=0.24153(12)$. 


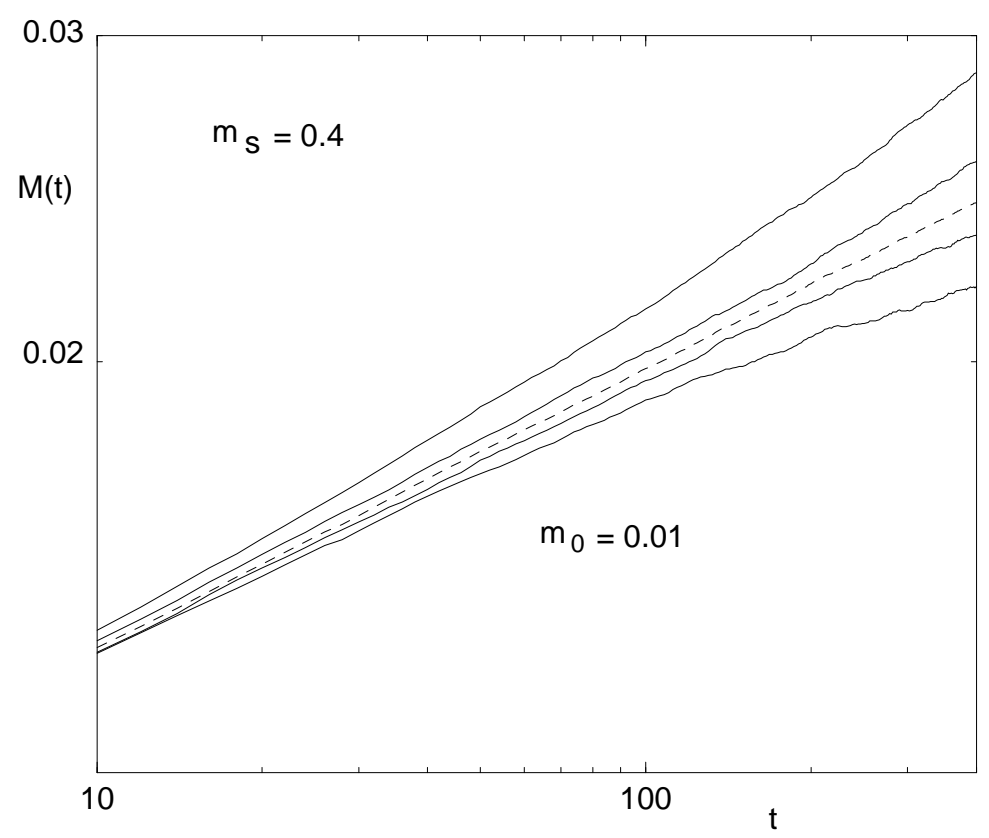

FIG. 6. Time evolution of the staggered magnetization for $m_{s}=0.4$ in log-log scale. For solid lines, $K$ is $0.3310,0.3320,0.333$ and 0.3340 (from below). The dashed line corresponds to $K_{c}=0.33230(20)$ 


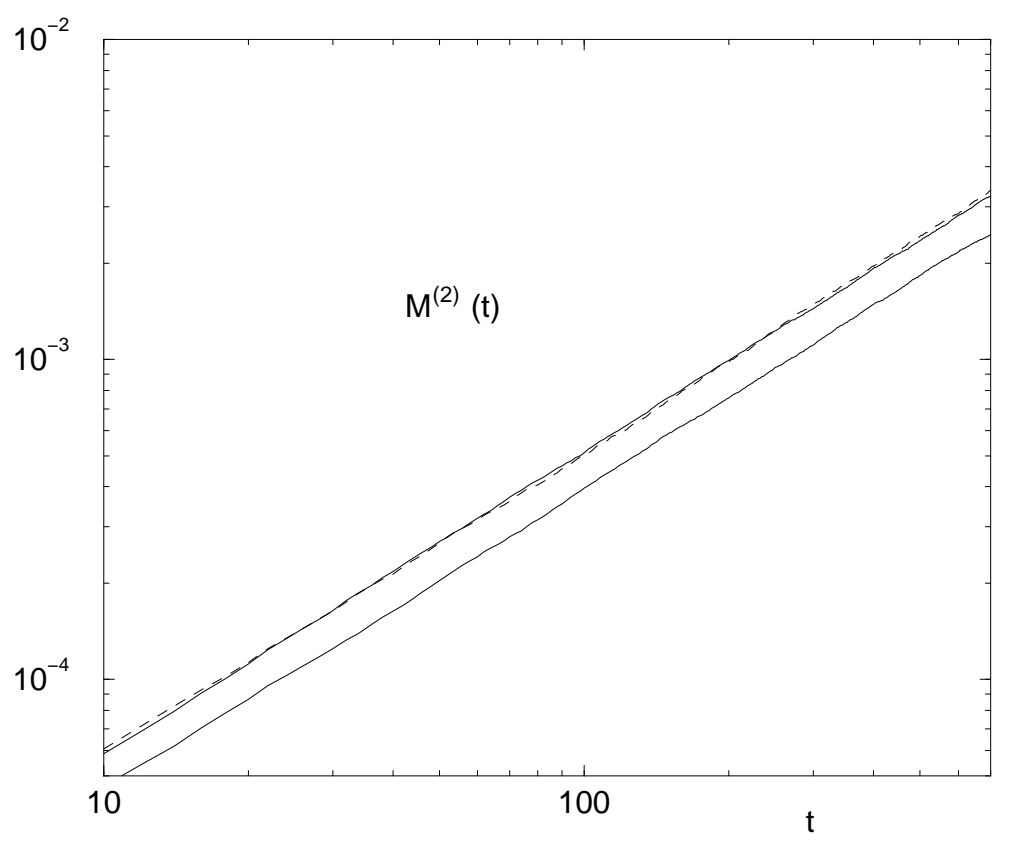

FIG. 7. The second moment at $K_{c}$ in log-log scale. For solid lines, $m_{s}=0.2$ and 0.4 (from above). The dashed line is for $m_{s}=0.0$. 


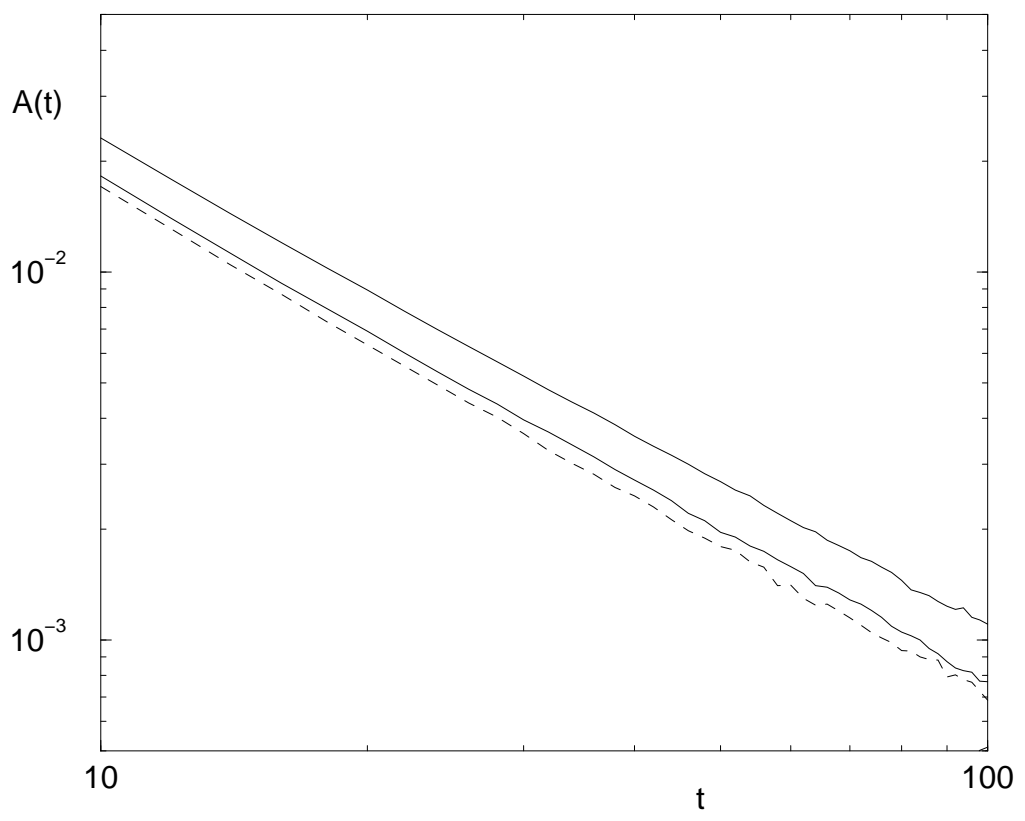

FIG. 8. The auto-correlation at $K_{c}$ in log-log scale. For solid lines, $m_{s}=0.2$ and 0.4 (from below). The dashed line is for $m_{s}=0.0$. 


\section{TABLES}

\begin{tabular}{l|lllll|lll}
\hline \hline$m_{s}$ & $K_{c}$ & $\theta$ & $\lambda$ & $y$ & $c_{d}$ & $z$ & $2 \beta / \nu$ & $\nu$ \\
\hline 0.0 & $0.22169(9)$ & $0.108(5)$ & $1.36(3)$ & $0.965(11)$ & $0.867(10)$ & $2.04(4)$ & $1.03(4)$ & $0.64(2)$ \\
\hline 0.2 & $0.24153(12)$ & $0.129(5)$ & $1.34(2)$ & $0.954(4)$ & $0.725(70)$ & $2.04(3)$ & $1.05(3)$ & $0.82(8)$ \\
\hline 0.4 & $0.33230(20)$ & $0.148(4)$ & $1.31(1)$ & $0.945(8)$ & $0.748(20)$ & $2.06(2)$ & $1.05(3)$ & $0.81(3)$ \\
\hline model A & $0.22170(4)$ & $0.108(2)$ & $1.36(2)$ & $0.970(11)$ & & $2.04(2)$ & $1.034(4)$ & $0.633(3)$ \\
\hline Ising & $0.221655(1)$ & & & & & & $1.037(3)$ & $0.630(1)$ \\
\hline \hline
\end{tabular}

TABLE I. Critical exponents measured for different conserved magnetization $m_{s}$ 's. The values for dynamics of model A are taken from Ref. [46]. Under the item 'Ising' are the values for the standard Ising model in equilibrium 45. 